\title{
Polarized microwave forward model simulations for tropical storm Fanoos
}

\author{
C Balaji ${ }^{1, *}$, M Deiveegan ${ }^{1}, \mathrm{~S}_{\text {P Venkateshan }}{ }^{1}$, R M Gairola ${ }^{2}$, \\ A SARKAR ${ }^{2}$ and V K AgARWAL ${ }^{2}$ \\ ${ }^{1}$ Department of Mechanical Engineering, Indian Institute of Technology Madras, Chennai 600 036, India. \\ ${ }^{2}$ Meteorology and Oceanography Group, Space Applications Centre, ISRO, Ahmedabad, India. \\ *e-mail: balaji@iitm.ac.in
}

In the present study, forward radiative transfer simulations are carried out for the tropical cyclone Fanoos that hit the coast off south India in December 2005. The in-house radiative transfer package used for this study employs the doubling and adding method to calculate radiances leaving the top of the one dimensional precipitating atmosphere. The particle drop size distribution is assumed to follow a modified gamma distribution in respect of the cloud liquid water and cloud ice water content. For precipitation, the Marshall-Palmer particle size distribution is used. All the hydrometeor particles are assumed to be spherical and Lorentz Mie theory is used to evaluate the interaction parameters like absorption, scattering coefficients and polarized scattering matrix. In order to validate the drop size distributions and interaction parameter calculations, the simulated brightness temperatures are compared with the TMI measured brightness temperatures for all the channels. For carrying out this exercise, vertical hydrometeors retrieved by TMI are used as input. The differences between simulated and measured brightness temperatures are found to be within $\pm 10 \%$. The maximum difference in the brightness temperatures between the present work and the Eddington model which the TRMM algorithm employs is about $4.5 \mathrm{~K}$. This may become significant when retrieval of precipitation is attempted by combining the forward model with a suitable retrieval strategy, under tropical conditions.

\section{Introduction}

Tropical cyclones are an important source of rainfall for agriculture and other water applications over the regions of subtropics and tropics. They occasionally turn out to be major disasters for human beings (Varma et al 2006). Accurate, quantitative assessment of rainfall and hydrometeor profiles within tropical cyclones over oceans is a difficult problem because direct measurements are almost impossible. Rainfall measurements from space based microwave sensors offer a solution to this limitation. The accuracy of the microwave remote sensing of precipitation systems largely depends on the forward radiative transfer model (Petty 2001), which are themselves based on some assumptions and hence are never perfect.

The Tropical Rainfall Measuring Mission (TRMM) satellite was designed to measure precipitation with high resolution (pixel size of $4 \mathrm{~km}$ at $85 \mathrm{GHz}$ ). The TRMM gives information about the vertical distribution of hydrometeors, surface rain, type of the observed rain (convective or stratiform) and no-rain conditions. There is a possibility of linking the TRMM Microwave Imager (TMI) to other radiometers and this offers several advantages because several satellite borne radiometers, with additional channels and resolutions similar to that of the TMI, are planned to be launched in the future. For example, the Indo-French mission Megha Tropiques (Aguttes et al 2000) will launch a

Keywords. Megha tropiques; microwave; radiative transfer; simulations; polarized; tropical storm; Fanoos. 
satellite carrying a Microwave Analysis and Detection of Rain and Atmospheric Systems (MADRAS) with radiometers similar to the TMI. The frequencies of the various channels are 18.7, 36.5, 89, $157 \mathrm{GHz}$ (all horizontal and vertical polarizations) and $23.8 \mathrm{GHz}$ (only vertical polarization).

There are different ways to validate the accuracy of any retrieval algorithm (Shige et al 2006). One such method that is frequently used for validation purposes is a comparison of the retrieved surface rainfall rate with ground truth. However, the validation need not be confined to surface rainfall measurements, which are only loosely related to the volumetric observations made by most sensors (Viltard et al 2000). One simple method will be to calculate the brightness temperatures using retrieved profiles of hydrometeors and to compare these with the measured brightness temperatures. In the present work, the TMI retrieved profiles are used to investigate the consistency between the model and the measurement. Instead of depending on the actual measured data for validation, the present work uses TMI retrieved profiles of hydrometores and the assumption of modified gamma distribution for the drop sizes to check the consistency. The differences between the computed and measured brightness temperatures are sensitive to the assumptions on microphysical properties, such as particle shape, density, and size. If the assumptions are incorrect, the inconsistency may or may not be evident in the form of unresolvable differences between the observed and computed multichannel brightness temperatures (Petty 2001). Some of the potential uncertainties associated with modeling microwave radiative transfer in rain clouds are addressed by (Bauer et al 2000).

The main objectives of this study are:

- to evaluate the performance of the forward model against TMI observations for the tropical storm Fanoos, and

- to compare the present simulations with the results from the Eddington solution.

\section{Forward model}

The atmosphere contains gaseous molecules (oxygen and water vapour), cloud (liquid and ice) and precipitation (rain and ice) particles. Microwave radiation from the atmosphere is due to the emission, absorption and scattering of these particles. To simulate the atmospheric radiation and retrieve the profiles of hydrometeors using radiometric measurements, an accurate and realistic forward model is needed.

The atmosphere is subdivided into several homogeneous horizontal layers, to each of which mean values for temperature and pressure are assigned.
For the problem under consideration, temperature, pressure and humidity values applicable to a raining atmosphere are taken from the Goddard Cloud Ensemble (GCE) profiles (Tao and Simpson 1993). Additionally, layer averaged values of hydrometeors for given layers are calculated from the input profiles. For different types of hydrometeors in a given layer, the governing characteristics such as size, density, type of single scattering calculations, type of drop size distribution, distribution parameters and the shape are also assigned. The computation of the single scattering properties (extinction, scattering albedo and phase matrix) for the population of hydrometeors present at each layer of the medium is an important step in the radiative transfer calculations. The total extinction and scattering coefficients due to gases, nonprecipitating cloud, and precipitation are combined based on Ulaby et al (1981). The water vapour absorption is calculated according to Ulaby et al (1981) and the gaseous absorption part is calculated based on Liebe et al (1985).

For liquid cloud, it is usually assumed that absorption dominates over scattering. The Rayleigh approximation is applicable for computing the absorption coefficients of cloud droplets. A review of literature shows that a small number of large cloud droplets can contribute significantly to the scattering even when the mode size of the particle size distribution is in the order of 10 to 100 micrometers (Zhang et al 1999). In this case, the Rayleigh scattering approximation is not valid and an exact solution must be used (Zhang et al 1999). In this study, rain, ice and cloud hydrometeors are assumed spherical in shape and the classical Lorenz-Mie theory (Wiscombe 1980) is applied for the calculation of absorption coefficient, scattering coefficient and the phase matrix (Evans and Stephens 1991). The refractive index of rain droplets, precipitation ice, cloud water particles are based on Ulaby et al (1981).

In this study, a group of water or ice particles is assumed to follow the size distribution given by the modified gamma function (Deirmendjian 1969). The modified gamma function is widely used in literature and other functions such as MarshallPalmer distribution (Marshall and Palmer 1948), turn out to be its special cases.

Clouds are homogeneous layers containing liquid, ice, or a mixed phase. The cloud droplet size distribution is an important quantity in determining cloud micro-physics and atmospheric radiation. The drop size distribution for either water or ice is given by the modified gamma function, with four parameters $a, b, \alpha$, and $\gamma$, as:

$$
n(r)=a r^{\alpha} \exp \left(-b r^{\gamma}\right)
$$


An alternative form of equation (1) is:

$$
n(r)=a r^{\alpha} \exp \left(-\left(\frac{\alpha}{\gamma}\right)\left(\frac{r}{r_{c}}\right)^{\gamma}\right),
$$

where the critical radius $r_{c}$ is given by:

$$
r_{c}=\left(\frac{\alpha}{\gamma b}\right)^{1 / \gamma} .
$$

The mass density $m$ (for liquid or solid) is given by:

$$
\begin{aligned}
m & =\int_{0}^{\infty}\left(\frac{4}{3} \pi r^{3}\right) \rho n(r) d r \\
& =a \rho\left(\frac{4 \pi}{3 \gamma}\right)\left(\frac{\alpha}{\gamma}\right)^{-(\alpha+4) / \gamma} r_{c}^{\alpha+4} \Gamma\left(\frac{\alpha+4}{\gamma}\right),
\end{aligned}
$$

where $\Gamma$ and $\rho$ represent the gamma distribution function and the density of the hydrometeor respectively. The equation for mass density is integrable for all possible values of the radius only when (i) $a, \gamma>0$ (ii) $b \geq 0$ and $\alpha>-4$. For $\alpha \leq 0, r_{c}$ is undefined and must be reinterpreted for use in equation (2), though equation (1) remains valid. When $\alpha=0$, the Marshall-Palmer distribution given by:

$$
n(r)=16 \times 10^{6} \exp \left(-8200 r R_{r}^{-0.21}\right) \quad \text { in } \mathrm{m}^{-4},
$$

is obtained, where $r_{c}$ is replaced now by $R_{r}$, the rain rate in $\mathrm{mm} / \mathrm{h}$, and the constant $b$ is adjusted to the value shown, in keeping with the experimental observations.

Parameter ' $a$ ' is the normalizing constant that is fixed by mass density in equation (4). The calculations are performed for a given set of $\alpha, \gamma$, and $r_{c}$ over the desired temperature and frequency ranges. The values of these constants are based on Ulaby et al (1981).

The ocean surface is characterized by sea surface temperature, salinity and wind speed. In the present work, specular surface approximations are used to model the sea surface. For a specular surface, the reflected radiation is polarized and angle dependent, and the bidirectional reflection matrix is given by (Tsang 1985): where the vertical and horizontal reflection coefficients are given by the Fresnel relations:

$$
\begin{aligned}
& R_{v}(\theta)=\frac{m^{2} \cos \theta-\sqrt{m^{2}+\cos ^{2} \theta-1}}{m^{2} \cos \theta+\sqrt{m^{2}+\cos ^{2} \theta-1}} \\
& R_{h}(\theta)=\frac{\cos \theta-\sqrt{m^{2}+\cos ^{2} \theta-1}}{\cos \theta+\sqrt{m^{2}+\cos ^{2} \theta-1}}
\end{aligned}
$$

and $m$ is the complex index of refraction of the surface that is also related to the complex dielectric constant. For sea water, the dielectric constant is complex and is a function of physical parameters (sea surface temperature and salinity) and the frequency of the electromagnetic wave. The dielectric constant model is based on Klein and Swift (1977). The energy emitted by a Fresnel surface is also polarized and angle dependent, and the emission vector is given by Evans and Stephens (1991):

$$
\bar{\varepsilon}=\left(\begin{array}{c}
1-\frac{1}{2}\left(\left|R_{v}\right|^{2}+\left|R_{h}\right|^{2}\right) \\
-\frac{1}{2}\left(\left|R_{v}\right|^{2}-\left|R_{h}\right|^{2}\right) \\
0 \\
0
\end{array}\right)
$$

The action of wind on the ocean surface leads to significant deviations of the reflectivity and surface emissivity from those predicted by Fresnel relations. The effect of sea surface roughness due to wind speed is incorporated in the model by using the formulation proposed by Wisler and Hollinger (1977). High winds cause wave breaking and roughness in the sea surface. The wave breaking, in turn, leads to the generation of foam. This foam coverage also affects the polarized emissivities of sea surface. In this study, foam coverage model has been implemented following the methodology adopted by Monahan and O' Muirchantaigh (1986). The total reflectivity is calculated by:

$$
\overline{\bar{R}}(\theta)=\left(\begin{array}{cccc}
\frac{1}{2}\left(\left|R_{v}\right|^{2}+\left|R_{h}\right|^{2}\right) & \frac{1}{2}\left(\left|R_{v}\right|^{2}-\left|R_{h}\right|^{2}\right) & 0 & 0 \\
\frac{1}{2}\left(\left|R_{v}\right|^{2}-\left|R_{h}\right|^{2}\right) & \frac{1}{2}\left(\left|R_{v}\right|^{2}+\left|R_{h}\right|^{2}\right) & 0 & 0 \\
0 & 0 & \operatorname{Re}\left(R_{v} R_{h}^{*}\right) & -\operatorname{Im}\left(R_{v} R_{h}^{*}\right) \\
0 & 0 & \operatorname{Im}\left(R_{v} R_{h}^{*}\right) & \operatorname{Re}\left(R_{v} R_{h}^{*}\right)
\end{array}\right)
$$




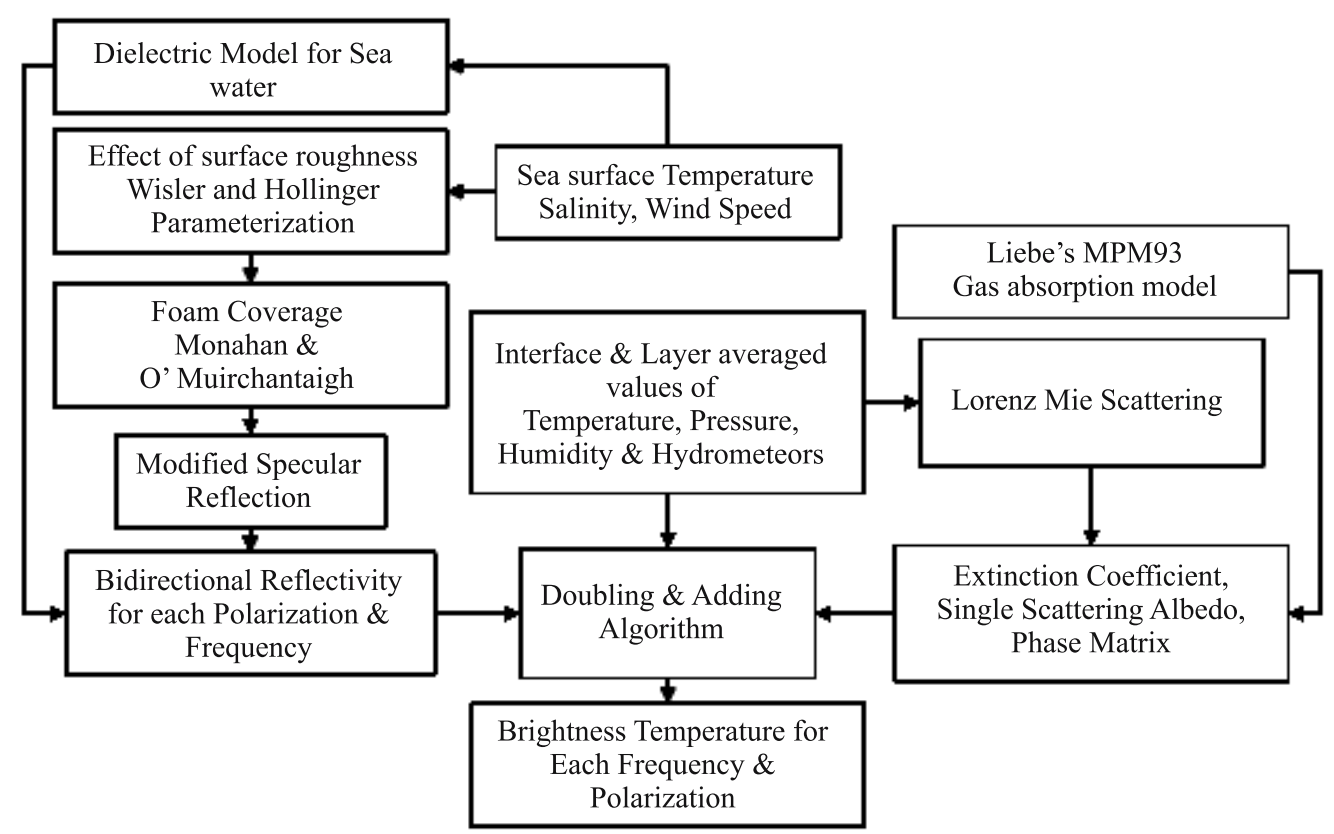

Figure 1. A bird's eye view of the microwave radiative transfer model.

- the sum of foam reflectivity weighted with the foam coverage, and

- the sum of water reflectivity weighted with water coverage.

The radiative transfer model gives the simulated brightness temperatures for each viewing angle and frequency, for a downward looking space borne radiometer. The plane parallel solution is sufficient to cover most applications for radiation scattering in planetary atmospheres. To compute polarized radiation in plane parallel atmosphere, the well known doubling-adding algorithm is used. The present work adopts the standard doubling-adding scheme as described by (Evans and Stephens 1991).

The radiative interaction parameters (surface reflectivities, extinction, scattering albedo and phase matrix) are given as input for the radiative transfer calculations. A detailed flow diagram indicating the various steps employed in the forward model is shown in figure 1. Following the standard formulation (Evans and Stephens 1991), the monochromatic plane parallel polarized radiative transfer equation for randomly oriented particles is written as:
Here $I$ is the diffuse radiance field expressed as the vector of four Stokes parameters $(I, Q, U, V), P$ is the $4 \times 4$ scattering (or Mueller) matrix, $B(T)$ is the Planck blackbody function, $\tilde{\omega}$ the single scattering albedo, $\tau$ the optical depth, $\mu$ the cosine of the zenith angle $\theta$ and $\phi$ the azimuth angle. The co-ordinate system is such that $\tau$ increases downwards and $\mu$ is positive for the lower hemisphere. The angular variation of radiation is written as a Fourier series in the azimuth direction and by discretization in the zenith angle using numerical Gaussian quadrature. The radiance at any position inside the atmosphere is represented by Stokes parameters, quadrature zenith angles and the Fourier azimuth modes. The radiance field is computed via the doubling-adding algorithm (see Deiveegan 2007 for a fuller discussion of the algorithm).

All the computations have been performed on a PC equipped with Intel-3.2 GHz, Pentium-4 processor, 4GB of RAM and Compaq Visual FORTRAN compiler. The average CPU time for simulation for one profile is around $3 \mathrm{~s}$.

$$
\mu \frac{d I}{d \tau}(\tau, \mu, \phi)=-I(\tau, \mu, \phi)+\frac{\tilde{\omega}}{4 \pi} \int_{0}^{2 \pi} \int_{-1}^{1} P(\mu, \phi ; \tilde{\mu}, \tilde{\phi}) d \tilde{\mu} d \tilde{\phi}+(1-\tilde{\omega}) B(T)\left[\begin{array}{l}
1 \\
0 \\
0 \\
0
\end{array}\right]
$$


Table 1. Interaction parameters of two-layer atmosphere at $85.5 \mathrm{GHz}$.

\begin{tabular}{lcccccccr}
\hline & \multicolumn{3}{c}{ Present work } & & \multicolumn{3}{c}{ Evans and Stephens (1991) } \\
\cline { 2 - 3 } Layer & Index of refraction & $\sigma_{e}\left(\mathrm{~km}^{-1}\right)$ & $\sigma_{s}$ & & Index of refraction & $\sigma_{e}\left(\mathrm{~km}^{-1}\right)$ & $\sigma_{s}$ \\
\hline 1 & $(1.7800,-0.00379)$ & 0.1613 & 0.3874 & & $(1.7829,-0.00344)$ & 0.1522 & 0.3818 \\
2 & $(3.2548,-1.9106)$ & 0.1649 & 0.9807 & & $(3.2781,-1.8512)$ & 0.1354 & 0.9819 \\
\hline
\end{tabular}

Table 2. Legendre coefficients of the phase matrix elements (present work).

\begin{tabular}{rrrrrrrr}
\hline & \multicolumn{3}{c}{ Rain } & & \multicolumn{3}{c}{ Ice } \\
\cline { 2 - 3 } \cline { 6 - 7 } $\mathrm{L}$ & $\alpha_{1}^{l}$ & $\alpha_{2}^{l}$ & $\alpha_{3}^{l}$ & & $\alpha_{1}^{l}$ & $\alpha_{2}^{l}$ & $\alpha_{3}^{l}$ \\
\hline 1 & 1.000000 & -0.375244 & 0.127736 & & 1.000000 & -0.189088 & 0.720956 \\
2 & 0.386944 & -0.087425 & 1.481324 & & 1.336266 & -0.111275 & 1.678288 \\
3 & 0.522261 & 0.347112 & 0.380398 & & 0.945860 & 0.161665 & 0.890541 \\
4 & 0.124524 & 0.082020 & 0.078486 & & 0.377109 & 0.096425 & 0.390952 \\
5 & 0.037523 & 0.027034 & 0.010850 & & 0.148557 & 0.025848 & 0.130421 \\
6 & 0.007375 & 0.005215 & 0.001499 & & 0.038373 & 0.013658 & 0.038483 \\
7 & 0.001484 & 0.001065 & 0.000130 & 0.014864 & 0.001544 & 0.011647 \\
8 & 0.000260 & 0.000185 & 0.000006 & 0.002494 & 0.001117 & 0.002712 \\
9 & 0.000045 & 0.000032 & -0.000002 & 0.001226 & 0.000040 & 0.000759 \\
10 & 0.000007 & 0.000005 & -0.000001 & 0.000121 & 0.000072 & 0.000165 \\
11 & & & & 0.000096 & -0.000007 & 0.000042 \\
12 & & & & 0.000005 & 0.000003 & 0.000010 \\
\hline
\end{tabular}

Table 3. Legendre coefficients of the phase matrix elements (Evans and Stephens).

\begin{tabular}{|c|c|c|c|c|c|c|}
\hline \multirow[b]{2}{*}{$\mathrm{L}$} & \multicolumn{3}{|c|}{ Rain } & \multicolumn{3}{|c|}{ Ice } \\
\hline & $\alpha_{1}^{l}$ & $\alpha_{2}^{l}$ & $\alpha_{3}^{l}$ & $\alpha_{1}^{l}$ & $\alpha_{2}^{l}$ & $\alpha_{3}^{l}$ \\
\hline 1 & 1.000000 & -0.378560 & 0.122149 & 1.000000 & -0.203665 & 0.706712 \\
\hline 2 & 0.365211 & -0.082115 & 1.482953 & 1.305650 & -0.111353 & 1.669173 \\
\hline 3 & 0.518055 & 0.353963 & 0.356369 & 0.915656 & 0.176185 & 0.856457 \\
\hline 4 & 0.115569 & 0.077666 & 0.067922 & 0.348084 & 0.097906 & 0.357357 \\
\hline 5 & 0.032699 & 0.023756 & 0.008445 & 0.131959 & 0.025961 & 0.114952 \\
\hline 6 & 0.006058 & 0.004312 & 0.001049 & 0.032286 & 0.012476 & 0.031836 \\
\hline 7 & 0.001139 & 0.000819 & 0.000071 & 0.011950 & 0.001494 & 0.009323 \\
\hline 8 & 0.000188 & 0.000133 & 0.000000 & 0.001923 & 0.000916 & 0.002046 \\
\hline 9 & 0.000030 & 0.000021 & -0.000002 & 0.000899 & 0.000030 & 0.000561 \\
\hline 10 & 0.000005 & 0.000003 & 0.000000 & 0.000085 & 0.000052 & 0.000112 \\
\hline 11 & & & & 0.000061 & -0.000004 & 0.000025 \\
\hline 12 & & & & 0.000003 & 0.000003 & 0.000006 \\
\hline 13 & & & & 0.000004 & -0.000001 & 0.000001 \\
\hline
\end{tabular}

\section{Results}

Since the forward model involves a combination of the interaction parameters calculations and the solution of the vector radiative transfer equations, it is necessary to check the accuracy of the model for various possible circumstances. In view of this, the present work is validated first against benchmark results, airborne measurements, ground based radiometer measurements and satellite data available in literature. The details of these extensive validations are available in Deiveegan (2007).

To verify the interaction parameter calculations for hydrometeors, the results of the present work are compared with the results of Evans and Stephens (1991), where the atmosphere is modeled 

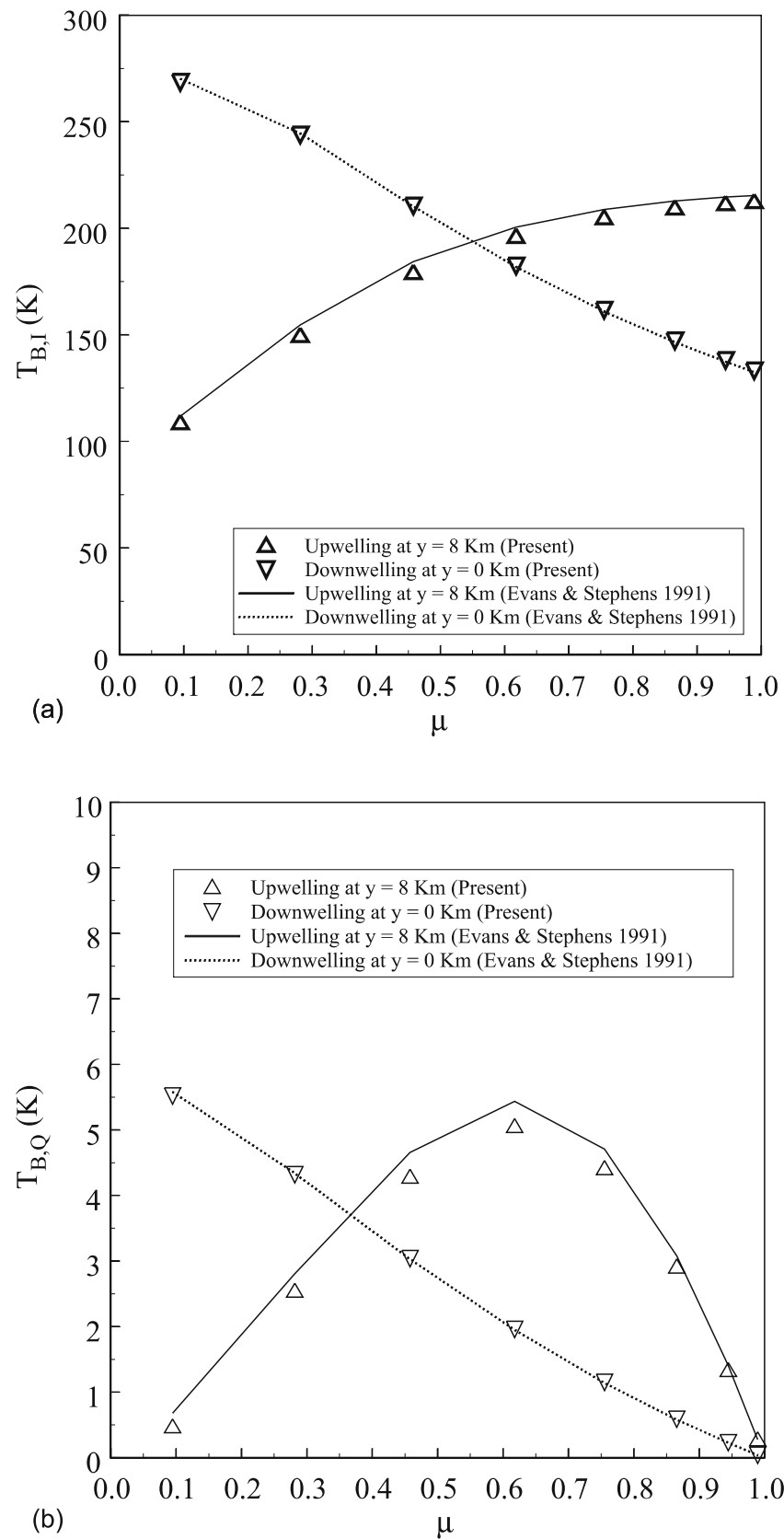

Figure 2. Comparison of Stokes parameters $I$ and $Q$ with literature for a case of two-layer atmosphere with a specular surface.

as a participating medium divided into two layers, the first layer being a rain layer and the second layer being an ice layer. The bottom surface is modeled as a diffuse surface with a reflectivity of $\rho_{0}=0.44$ and an emissivity of $\varepsilon=\left(1-\rho_{0}\right)=0.56$. Unpolarized, diffuse cosmic background radiation at a blackbody equivalent temperature of $2.7 \mathrm{~K}$ is assumed to be incident over the top of the atmosphere. The temperatures of the atmosphere are assumed to be $300 \mathrm{~K}$ at the bottom surface, $273 \mathrm{~K}$ at the rain ice interface and $245 \mathrm{~K}$ at top of the ice layer. Both layers have a Marshall-Palmer size distribution of spherical particles with the rain rate within the rain layer being $2 \mathrm{~mm} / \mathrm{h}$ and the ice content within the ice layer being $0.5 \mathrm{~mm} / \mathrm{h}$. The maximum particle diameter within each layer is $1 \mathrm{~cm}$. The frequency considered in this study is 85.5 GHz.

The interaction parameters (extinction coefficient and single scattering albedo) for each layer are shown in table 1 . The Legendre coefficients of phase matrix are given in tables 2 and 3 . The interaction parameters compare satisfactorily with the benchmark results.

As a consequence of the assumption that the atmosphere is plane parallel with only thermal sources of radiation, the $U$ and $V$ components of the Stokes vector are zero. In this case, the effect of polarization is only due to the scattering by spherical particles. Figure 2 shows a comparison of the radiances $I$ and $Q$ for various $\mu$ values obtained from the present work with the results of Evans and Stephens (1991). The variation of both the upwelling and downwelling intensities as a function of $\mu$ are presented in figure 2. It can be observed that the results compare satisfactorily with the benchmark results. The results show that the spherical particles of rain and ice slightly polarize the signal that is leaving the diffuse surface.

The goal of this paper is to evaluate the performance of the forward model for an extreme event such as tropical storm. The specific storm under consideration is Fanoos that made its landfall on the southeast coast of India on 9 December 2005. The input hydrometeor profiles required for the forward radiative transfer calculations are the TRMM's Microwave Imager (TMI) derived profiles. The TMI observation in this study is restricted to an area located between $3.79^{\circ} \mathrm{N}$ and $16.7^{\circ} \mathrm{N}$ in the north-south direction and between $81.1^{\circ} \mathrm{E}$ and $94.5^{\circ} \mathrm{E}$ in the eastwest direction at 5:02 UTC (coordinated Universal Time) on 6 December 2005. A comparison of the measured and simulated brightness temperatures will throw light on the consistency of the forward model with respect to the observations.

The surface rainfall distribution in the tropical storm (Fanoos) as observed from the PR and TMI are shown in figure 3. The TRMM profiles of rain, precipitation ice, cloud water and cloud ice are given as input to the forward model. The brightness temperatures depend on the sea surface characteristics and the atmospheric constituents. In this study, the sea surface temperature for (north Indian Ocean) is assumed to be $300 \mathrm{~K}$; the salinity and wind speed are set to constant values of $35 \mathrm{ppm}$ and $15 \mathrm{~m} / \mathrm{s}$ respectively. Simulations were also performed using actual gridded wind speed data from QSCAT. For the higher frequency channel $(85 \mathrm{GHz})$, the differences in the brightness 


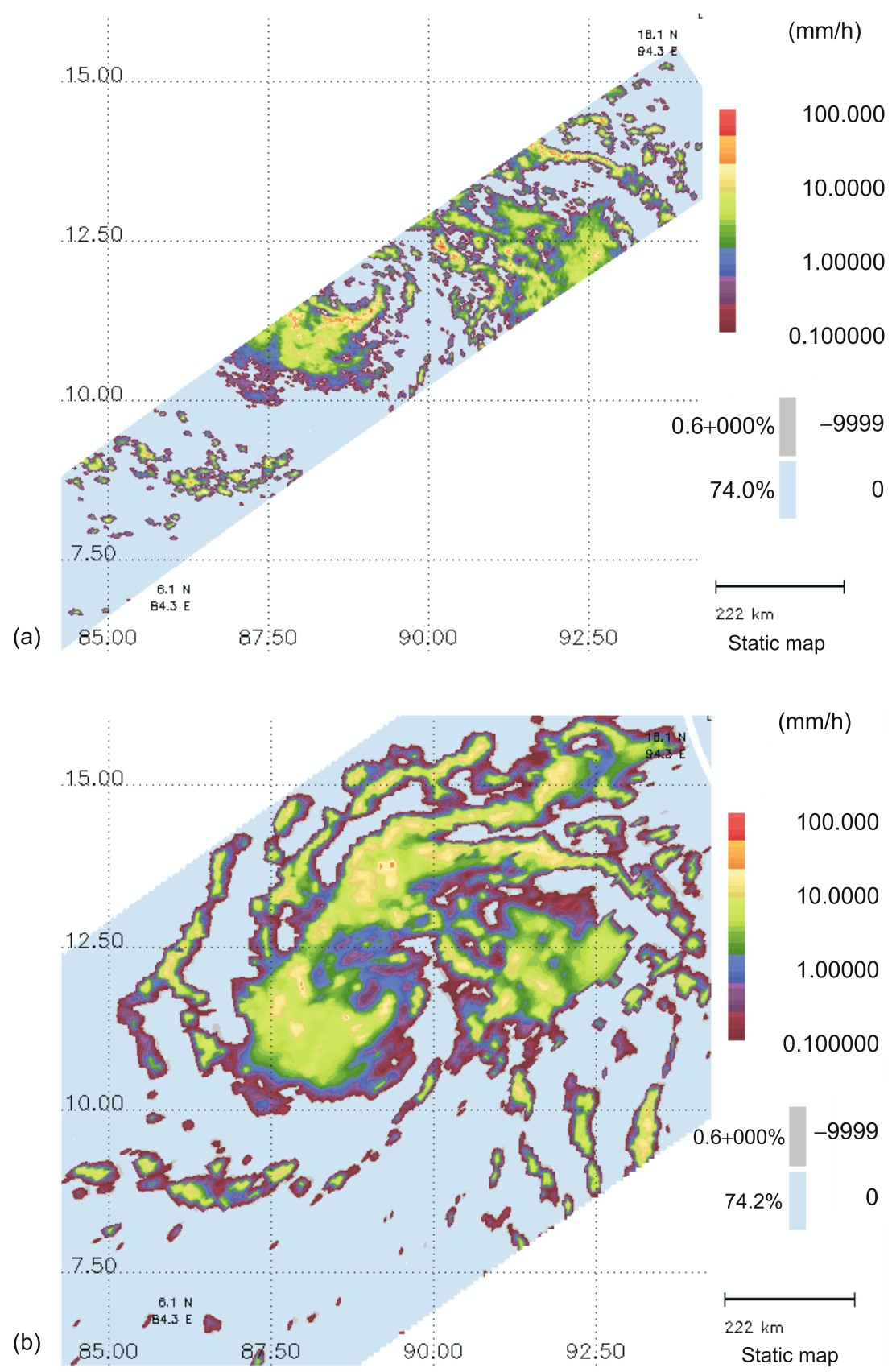

Figure 3. Surface rain rate measured by (a) PR and (b) TMI for the tropical storm Fanoos on 6 December 2005.

temperatures between the two simulations were found to be insignificant $(<0.2 \%)$.

The TMI measured and brightness temperatures simulated in this study for the $85 \mathrm{GHz}$ channel for both the vertical and horizontal polarizations are shown in figure 4 . The $85 \mathrm{GHz}$ channel is more sensitive to ice scattering and will serve as a good 'test' for the methodology adopted for handling scattering in the radiative transfer model. It is pertinent to mention here that simulations are carried out only for raining pixels over the ocean. Pixels over Sri Lanka have not been included for the simulations. Even so, in order to have one to one correspondence with the TRMM footprint, simulation results have been shown for the same footprint as the TRMM. However, it is worth mentioning that in all the plots, only the TRMM measurements show some signature over Sri Lanka. Our simulations set the temperatures over all pixels in the Sri Lankan region to values corresponding to the clear pixel over the Bay of Bengal.

One may notice that the agreement is generally good between the observed and the simulated brightness temperatures. The general pattern of tropical storm is very well reproduced at $85 \mathrm{GHz}$ and the eye wall region and rain bands can be 

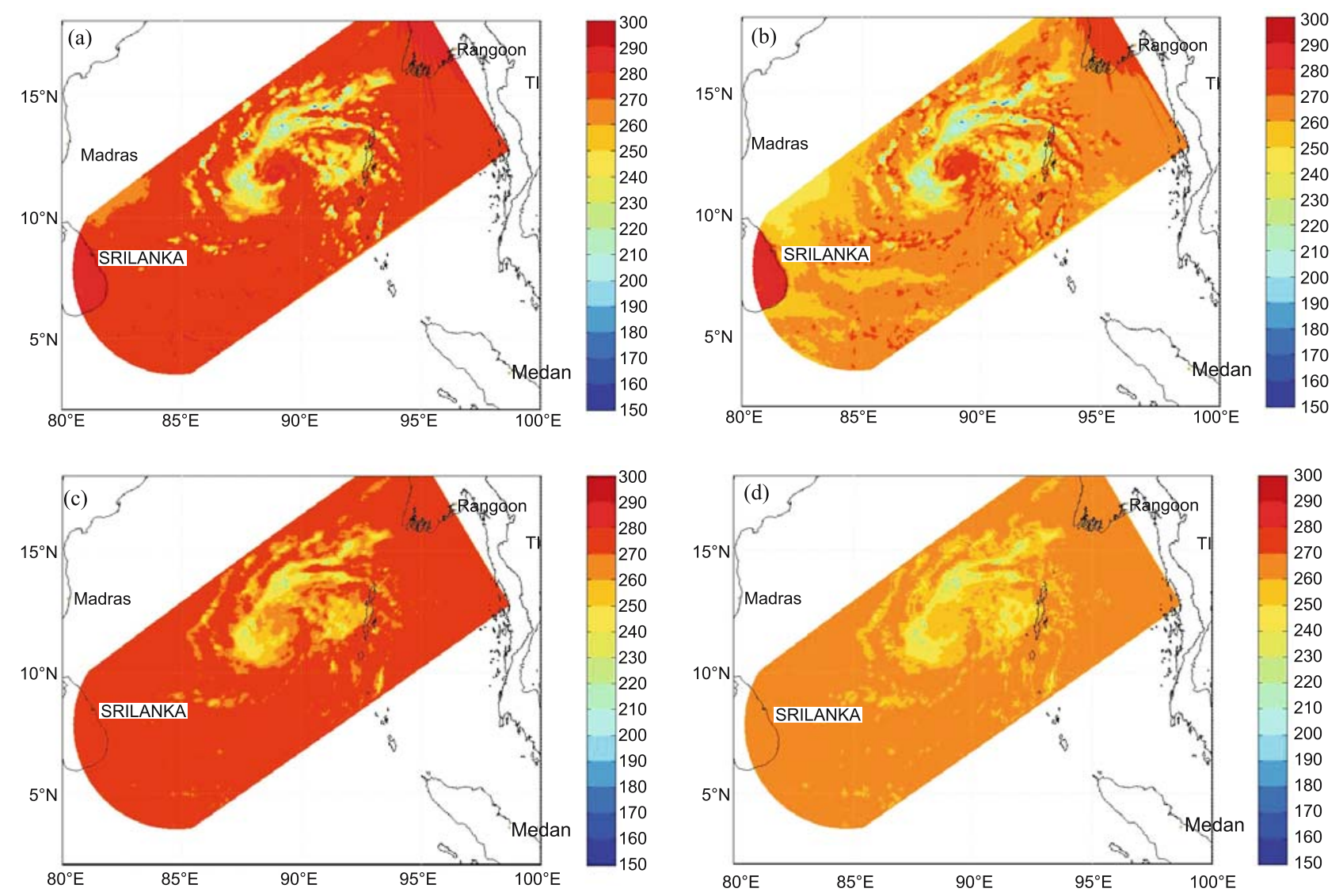

Figure 4. Brightness temperatures at $85 \mathrm{GHz}$ (a) TRMM (vertical polarization), (b) TRMM (horizontal polarization), (c) simulated (vertical polarization), and (d) simulated (horizontal polarization).

Table 4. Root-mean-square difference between simulated and observed brightness temperatures (85 GHz channel).

\begin{tabular}{|c|c|c|c|c|c|}
\hline \multirow{2}{*}{$\begin{array}{l}\text { Rain rate, } \\
\mathrm{mm} / \mathrm{h}\end{array}$} & \multirow{2}{*}{$\begin{array}{l}\text { No. of } \\
\text { data } \\
\text { points }\end{array}$} & \multicolumn{2}{|c|}{ RMS error, K } & \multicolumn{2}{|c|}{ RMS error, \% } \\
\hline & & $\mathrm{V}$ pol & $\mathrm{H} \mathrm{pol}$ & $\mathrm{V}$ pol & $\mathrm{H} \mathrm{pol}$ \\
\hline $0-5$ & 5447 & 10.17 & 12.82 & 4.09 & 5.12 \\
\hline $5-10$ & 1059 & 18.25 & 18.77 & 8.50 & 8.77 \\
\hline $10-15$ & 265 & 18.95 & 19.12 & 9.38 & 9.52 \\
\hline $15-20$ & 44 & 18.16 & 18.34 & 8.74 & 8.86 \\
\hline $0-20$ & 6815 & 12.27 & 14.23 & 5.34 & 6.08 \\
\hline
\end{tabular}

clearly seen. The computed brightness temperatures are close to the observations: the maximum difference is roughly $30 \mathrm{~K}$, which represents an error of about $10 \%$. Table 4 shows the root-meansquare difference between the observed and simulated brightness temperature for various rain rates. The error between simulated and observed brightness temperatures is less than $10 \%$. The differences are due to the following reasons:

- the hydrometeor profiles themselves are parameters that have been retrieved from TRMM brightness temperatures;

- melting layer has not been accounted in the forward model;

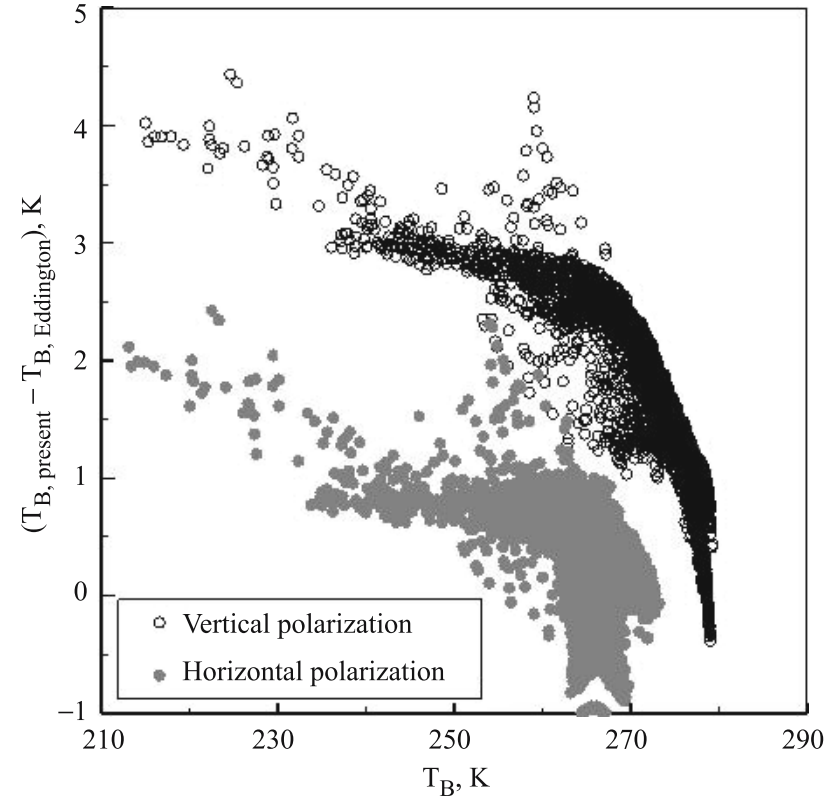

Figure 5. Differences between the simulated brightness temperatures at $85 \mathrm{GHz}$ using present work and the Eddington model.

- the hydrometeor particles are assumed to be spherical in shape; and

- effects of beam filling and geometry. 

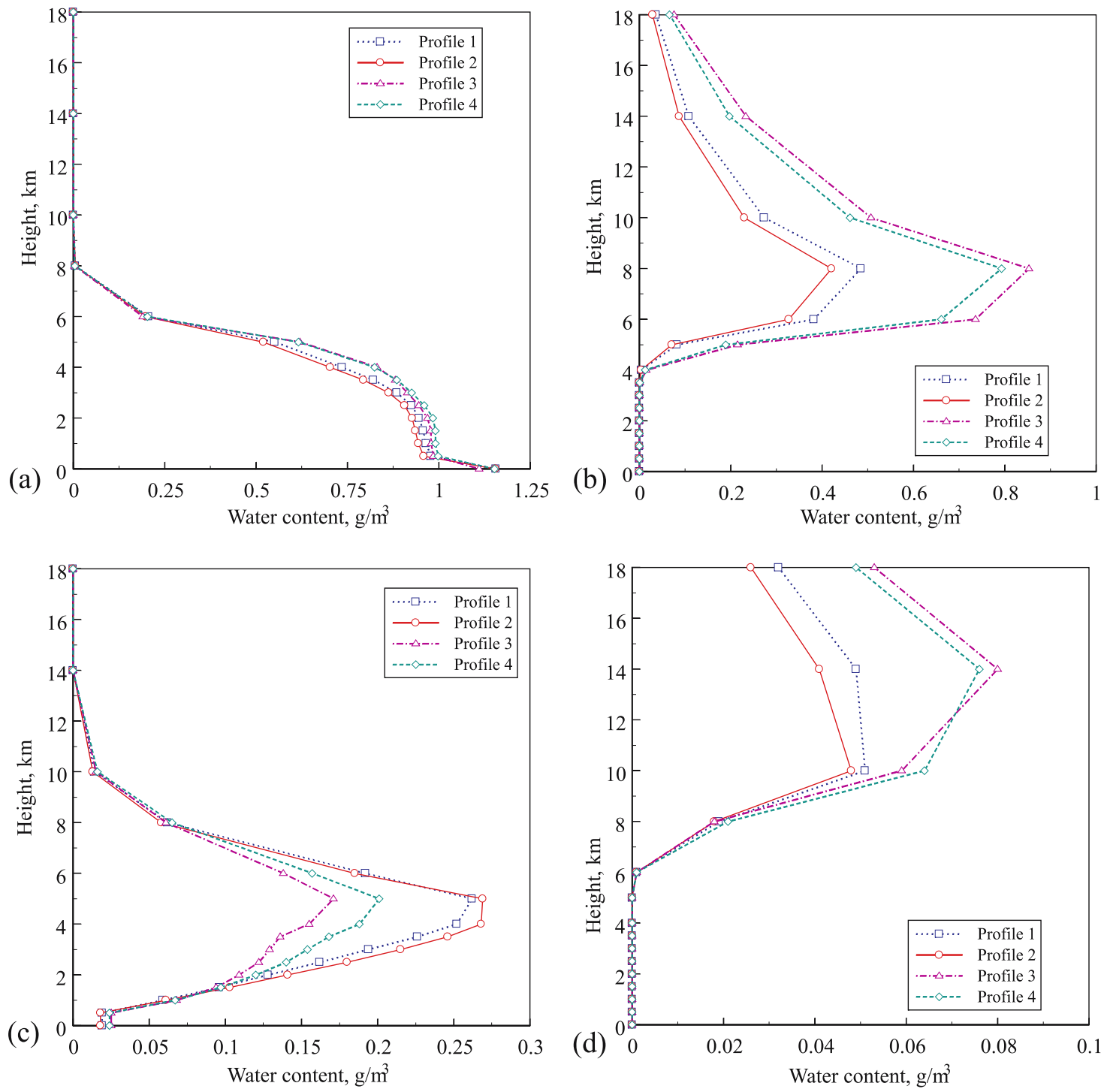

Figure 6. Input hydrometeor profiles (a) rain, (b) precipitation ice, (c) cloud liquid water, and (d) cloud ice water.

Table 5. Simulated and observed brightness temperatures for example profiles (85 GHz channel).

\begin{tabular}{|c|c|c|c|c|c|c|c|}
\hline \multirow[b]{2}{*}{ Profile } & \multirow{2}{*}{$\begin{array}{l}\text { Rain rate, } \\
\mathrm{mm} / \mathrm{h}\end{array}$} & \multicolumn{2}{|c|}{$T_{\mathrm{B}, \mathrm{TRMM}}, \mathrm{K}$} & \multicolumn{2}{|c|}{$T_{\mathrm{B}, \text { Simulated }}, \mathrm{K}$} & \multicolumn{2}{|c|}{$\begin{array}{c}\text { Difference, } \mathrm{K} \\
T_{\mathrm{B}, \mathrm{TRMM}}-T_{\mathrm{B}, \mathrm{Sim}}\end{array}$} \\
\hline & & $\mathrm{V} \mathrm{pol}$ & $\mathrm{H} \mathrm{pol}$ & $\mathrm{V}$ pol & $\mathrm{H} \mathrm{pol}$ & $\mathrm{V}$ pol & $\mathrm{H}$ pol \\
\hline 1 & 19.98 & 268.3 & 265.9 & 236.2 & 233.9 & 32.1 & 32.1 \\
\hline 2 & 19.97 & 268.7 & 268.3 & 240.0 & 237.7 & 28.7 & 30.6 \\
\hline 3 & 19.04 & 220.5 & 218.3 & 218.9 & 216.6 & 1.6 & 1.7 \\
\hline 4 & 19.91 & 228.6 & 227.2 & 221.7 & 219.4 & 6.9 & 7.8 \\
\hline
\end{tabular}

Another important reason for the differences is that for low rain rates, emission signature is significant vis-à-vis the scattering signature. The present study assumes GCE profiles for temperature, pressure and humidity, applicable for a rainy atmosphere. However, this becomes inevitable in the absence of reliable aircraft measurements of these quantities for a cyclonic storm over the north Indian Ocean. In view of this, some differences between TRMM measurements and simulated brightness temperatures are bound to occur.

Over and above all these, the ultimate aim of developing a forward model is to perform retrievals of geophysical parameters, the most important of which is the ground rain rate. Hence, even if there is a bias in the simulations with respect to the 


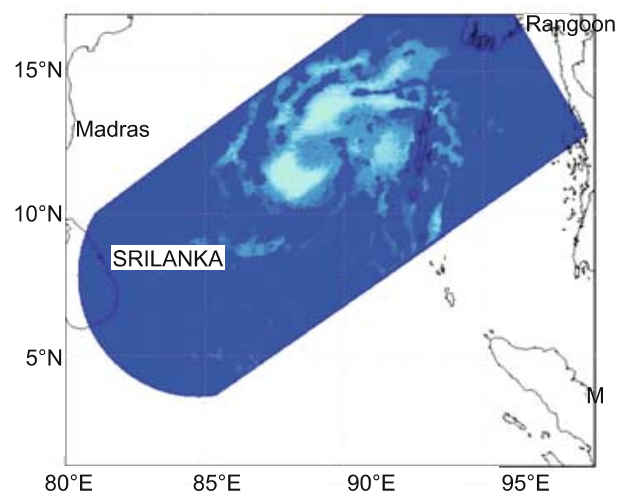

a) Simulated $T_{B}$ at $10 \mathrm{GHz}$, Vertical

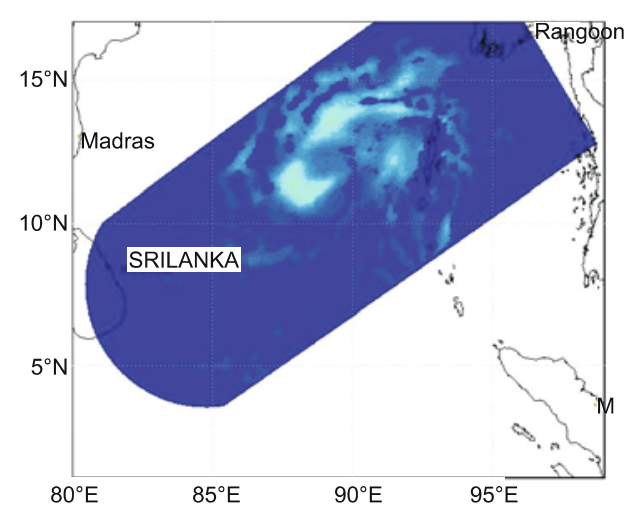

c) Simulated $T_{B}$ at $10 \mathrm{GHz}$, Horizontal

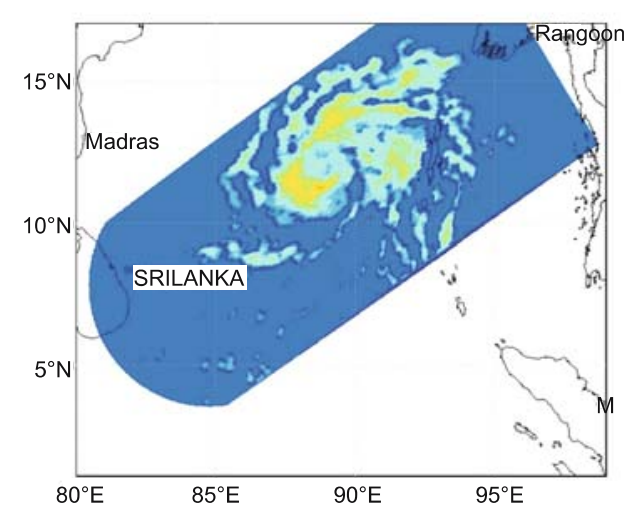

e) Simulated $T_{B}$ at $19 \mathrm{GHz}$, Vertical

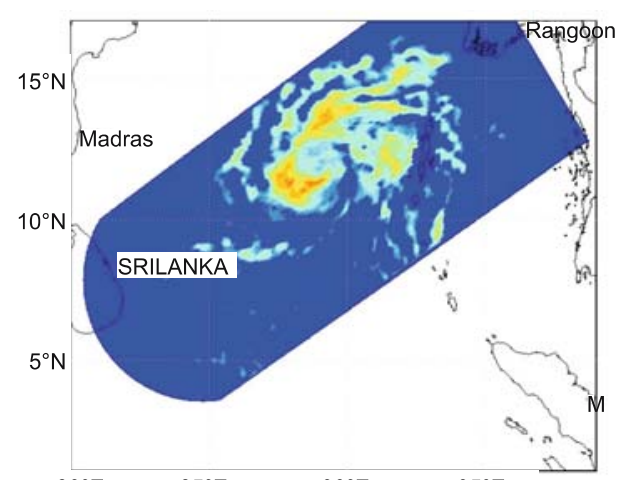

$80^{\circ} \mathrm{E} \quad 85^{\circ} \mathrm{E} \quad 90^{\circ} \mathrm{E} \quad 95^{\circ} \mathrm{E}$

g) Simulated $\mathrm{T}_{\mathrm{B}}$ at $19 \mathrm{GHz}$, Horizontal

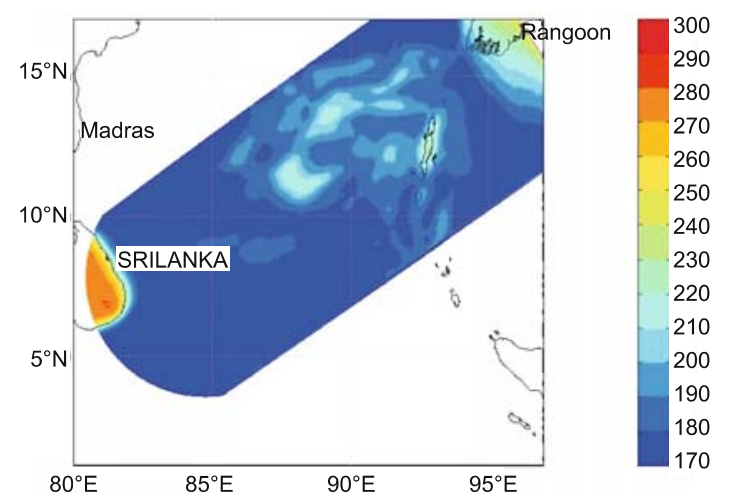

b) TRMM $T_{B}$ at $10 \mathrm{GHz}$, Vertical
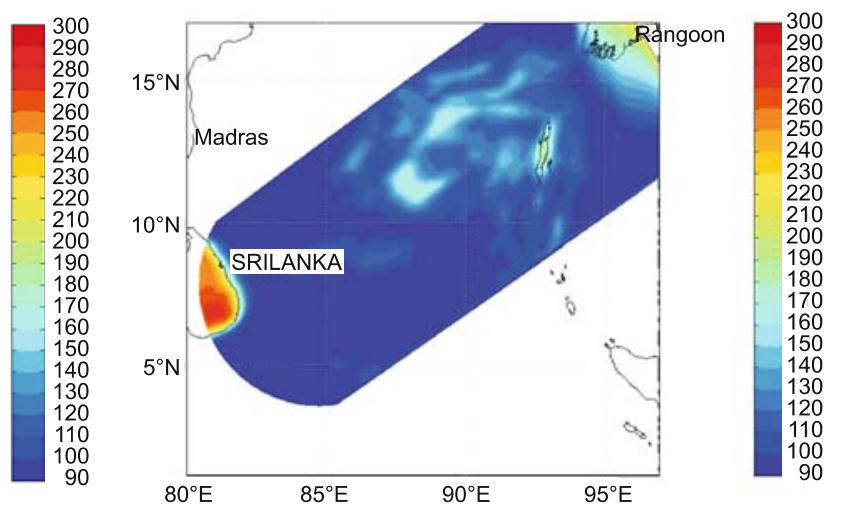

d) TRMM $\mathrm{T}_{\mathrm{B}}$ at $10 \mathrm{GHz}$, Horizontal
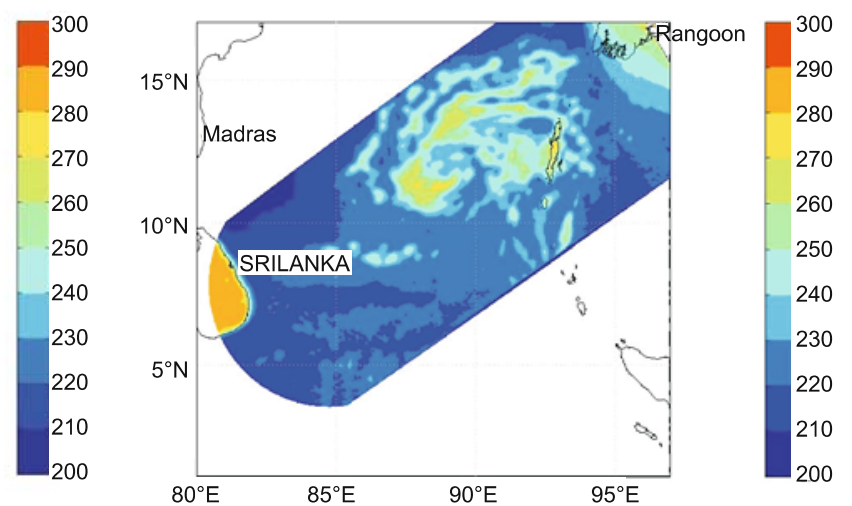

f) $\mathrm{TRMM} \mathrm{T}_{\mathrm{B}}$ at $19 \mathrm{GHz}$, Vertical
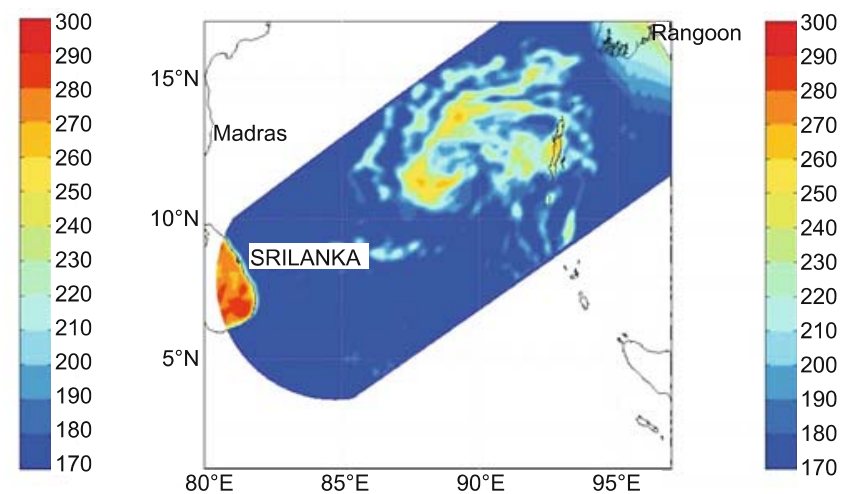

h) TRMM $T_{B}$ at $19 \mathrm{GHz}$, Horizontal

Figure 7. Comparison between brightness temperature simulated and TRMM-TMI. 


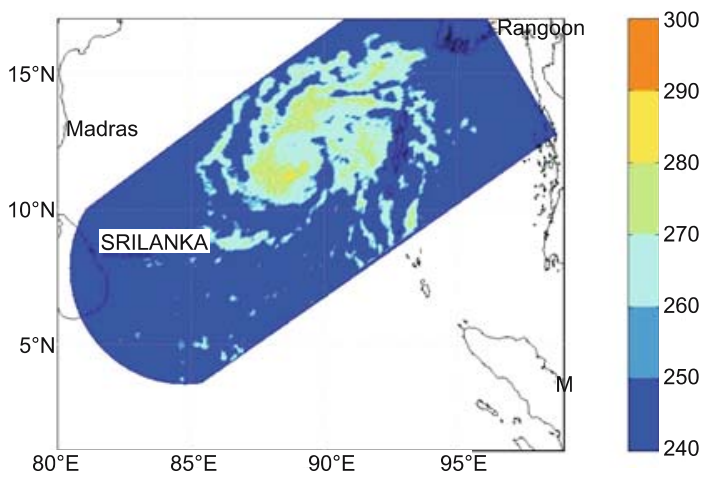

i) Simulated $T_{B}$ at $21 \mathrm{GHz}$, Vertical

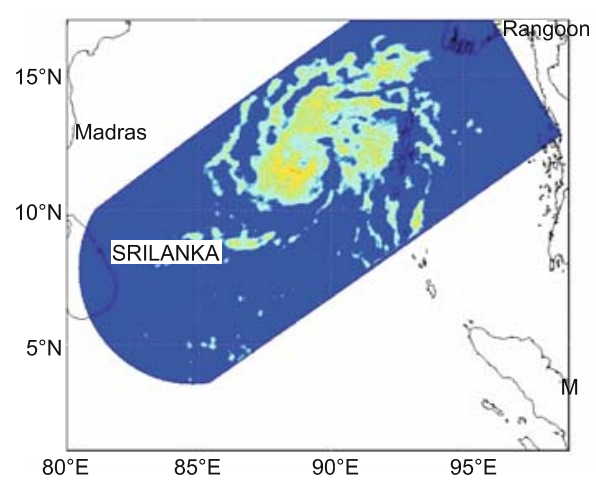

k) Simulated $T_{B}$ at $36 \mathrm{GHz}$, Vertical

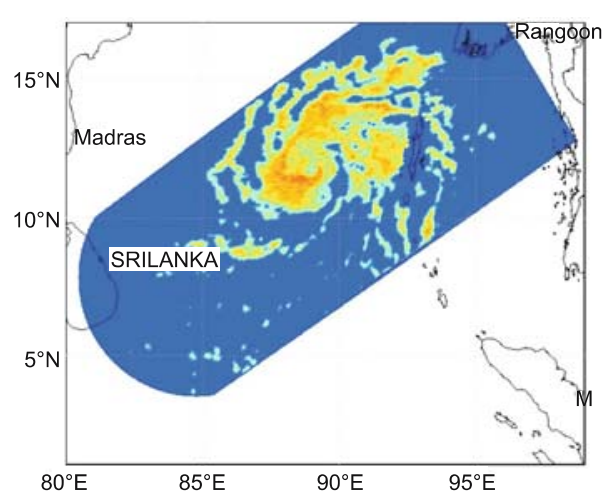

m) Simulated $T_{B}$ at $36 \mathrm{GHz}$, Horizontal

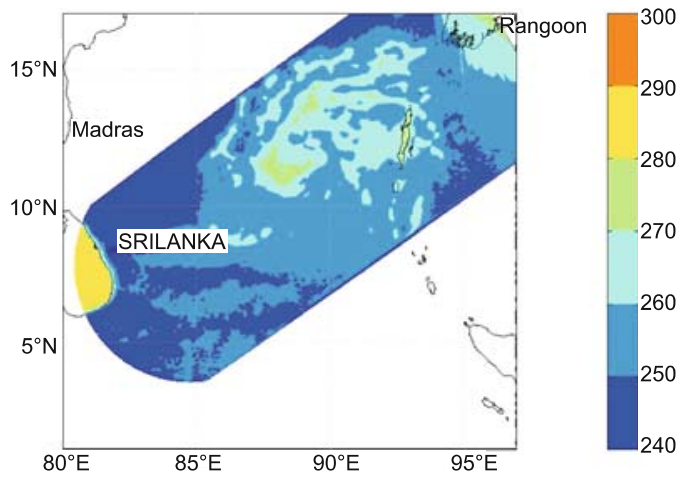

j) TRMM $T_{B}$ at $21 \mathrm{GHz}$, Vertical

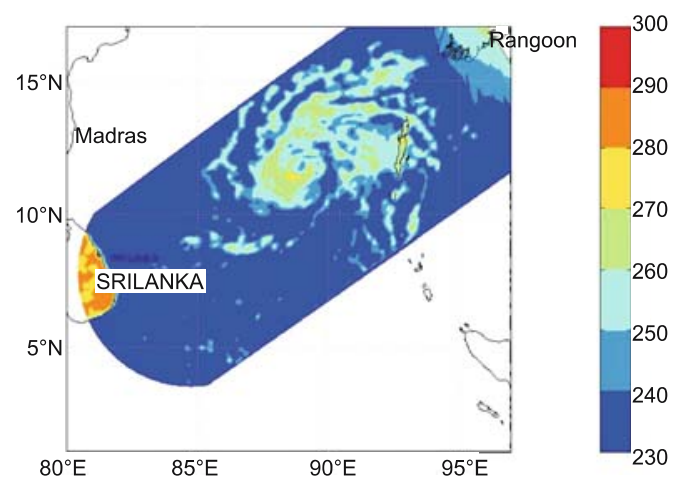

1) TRMM $T_{B}$ at $36 \mathrm{GHz}$, Vertical
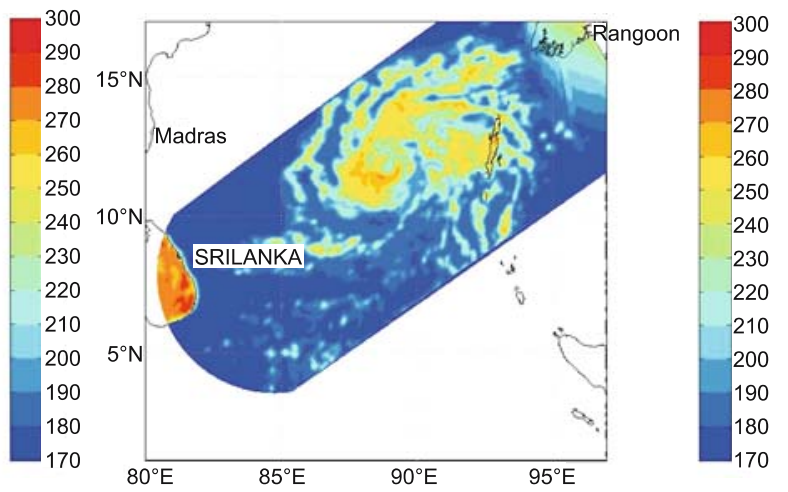

n) TRMM T $T_{B}$ at $36 \mathrm{GHz}$, Horizontal

Figure 7. (Continued).

measurements, since one seeks to specifically correlate, the difference in brightness temperature between a clear pixel and a rainy pixel with the rain rate rather than the brightness temperature itself to remove any bias, the differences between the TRMM measurements and simulations, are not expected to significantly affect the quality of the results. This is further corroborated by the fact that the simulations also show a consistent trend of decreasing brightness temperatures with increasing rain for this channel $(85 \mathrm{GHz})$, as already discussed.

Even though comparisons between the simulations of the present study and direct satellite measurements are satisfactory, it is important that further tests are required. For example, with the TRMM profiles as the input, if the Eddington model, on which the TRMM algorithm works is run and these results are compared with the simulations of this study, it will subdue the criticism that figure 4 indirectly validates only the TRMM retrieval. Hence, in order to check the consistency between models, the simulations have also been performed using the Eddington model (Kummerow 1996) for the tropical storm Fanoos. Accordingly, the brightness temperatures at $85 \mathrm{GHz}$ of Eddington model are compared with the results of present model (figure 5). The brightness temperatures 
simulated by both models agree very well and are within $5 \mathrm{~K}$. For low rain rates, when the brightness temperature are high the two models are indistinguishable, as expected. Thus, the polarization contribution by spherical hydrometeors can contribute up to $5 \mathrm{~K}$ which is significant. Even so, brightness temperatures observed by the TMI show that the effect of polarization due to nonspherical particles is significant. The present model offers the possibility of treating hydrometeors as nonspherical particles and hence will be less restrictive.

In order to demonstrate the effect of vertical structure of the hydrometeors on the brightness temperature, four TMI retrieved profiles (figure 6) having almost the same ground rain rate have been considered as inputs to the forward radiative transfer model. A comparison of the observed and computed brightness temperatures for the four hydrometeor profiles are shown in table 5 . The results show that for first two profiles the difference between the observed and simulated brightness temperature is large. However, for the last two profiles the model shows very good agreement with the observed profiles. In view of greater ice scattering, the brightness temperatures for the third and fourth profiles are less than for the first two profiles. This shows the poor correlation between the surface rainfall and the upper level ice amounts. This is due to the beam filling problem caused by the horizontal inhomogeneity of hydrometeors inside the satellite field of view. In order to check the validity of the present model for lower frequency channels, simulations are performed for these channels and the results are compared (figure 7) with TMI measured brightness temperatures. The comparison shows that the present model gives brightness temperatures close to TMI measurement for lower frequencies too.

The higher frequency channels play an important role in the retrieval of ice phase hydrometores. In MEGHA TROPIQUES higher frequency channels of $89 \mathrm{GHz}$ and $157 \mathrm{GHz}$ will be available, which may be used for the purpose. Finally, simulations are performed to identify the effect of atmospheric parameters on brightness temperatures for $157 \mathrm{GHz}$ (figure 8) to be used in the proposed Indo-French low orbiting, climate research satellite Megha Tropiques (Srinivasan 2006).

The brightness temperature is a highly nonlinear function of rain rate. For low frequencies $(18.7,23.8$, and $36.5 \mathrm{GHz})$, rainfall is characterized by warmer brightness temperature and is associated with the emission of rain drops. The rain cloud emits more radiation at lower frequencies than the background ocean and the cloud-free atmosphere. As a result, the brightness temperatures increase with rainfall. In these frequencies, rain affects the brightness temperature
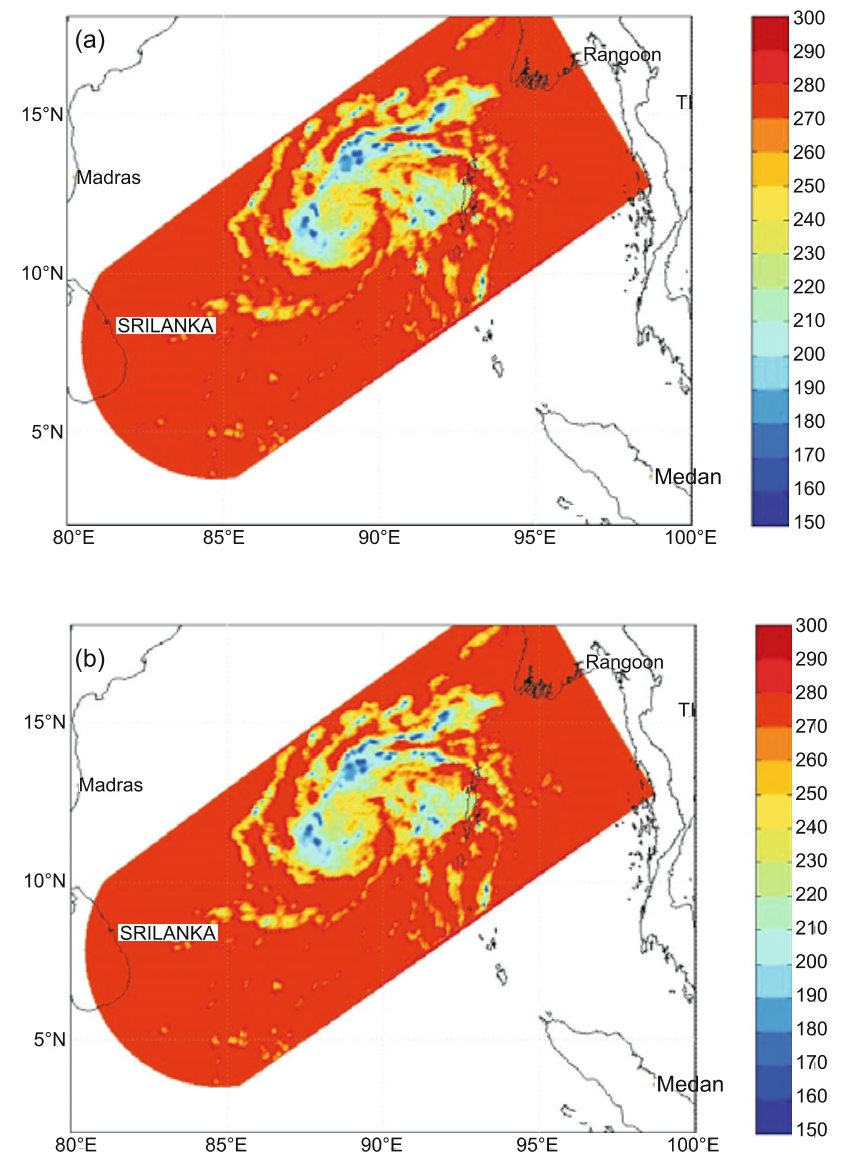

Figure 8. Brightness at $157 \mathrm{GHz}$ (a) simulated, vertical polarization and (b) simulated, horizontal polarization.

predominantly through emission and absorption. The scattering contributions are small. The signals are highly polarized at lower rain rate because of the specularly reflecting ocean viewed at an oblique angle. The signals further become unpolarized at higher rain rates because of randomly polarized emissions produced by raindrops. The brightness temperatures for both vertical and horizontal frequencies converge to saturation level for higher rain rate. Even in the lower frequency channels and at higher rain rates, the effect of scattering is evident, with a significant decay in brightness temperature. For higher frequency channels (89 and $157 \mathrm{GHz}$ ) scattering by rain and ice particles dominate, as expected. The brightness temperatures decrease rapidly. The differences in brightness temperatures for vertical and horizontal polarizations are very small compared to lower frequency channels. At lower rain rates, the signal becomes unpolarized due to scattering by rain and ice particles.

\section{Conclusions}

Forward radiative transfer simulations are carried out for the tropical storm Fanoos with an in-house code to validate 
- the retrieval ready forward model that consider polarization from both hydrometeors and the ocean

- modified drop size distribution for tropical conditions.

The one dimensional radiative transfer model includes the generation of interaction parameters to calculate the radiances leaving the top of the atmosphere. The doubling-adding algorithm is used to solve for the polarized intensity field, for a plane parallel atmosphere. All the hydrometeors are assumed to be spherical and the Lorenz-Mie theory is used to derive the extinction, scattering and phase matrices. Additionally, generalized modified gamma distributions are adopted to calculate single scattering properties for groups of hydrometer particles. Specifically, the Marshall-Palmer drop size distribution is used for rain and ice. The oceanic surface is assumed to be specular and uses the Fresnel model including correction for wind and foam. The simulated brightness temperatures show good agreement with the TMI brightness temperatures at $85 \mathrm{GHz}$, the channel sensitive to scattering. The average difference is roughly $12-15 \mathrm{~K}$, which represents an error of some $10 \%$. A very interesting conclusion of this study is that the widely used Eddington solution that ignores polarization from hydrometeors is reasonably accurate (difference between the fully polarized model and the Eddington model is within $5 \mathrm{~K}$ ).

The bias between the simulations and the TMI are likely due to the effect of melting layer and nonspherical nature of hydrometeor particles. Improvements like treatment of melting layer and non-spherical hydrometeors are in progress.

\section{References}

Aguttes J P, Schrive J, Goldstein C, Rouze M and Raju G 2000 Megha-Tropiques, a satellite for studying the water cycle and energy exchanges in the Tropiques; IEEE Geosci. Remote Sens. Symp. 7 3042-3044.

Bauer P, Khain A, Pokrovsky A, Meneghini R, Kummerow C, Marzano F and Poiares Baptista J P V 2000 Combined cloud-microwave radiative transfer modeling of stratiform rainfall; J. Atmos. Sci. $\quad \mathbf{5 7}$ 1082-1104.

Deirmendjian D 1969 Scattering on Spherical Polydispersion (New York, NY: Elsevier).

Deiveegan M 2007 Precipitation Retrieval using a Polarized Microwave Radiative Transport Model, Ph.D Thesis,
Department of Mechanical Engineering, Indian Institute of Technology Madras, India.

Evans K F and Stephens G L 1991 A new polarized atmospheric radiative transfer model; J. Quantitative Spectroscopy and Radiative Transfer 46 413-423.

Klein L A and Swift C T 1977 An improved model for the dielectric constant of sea water at microwave frequencies; IEEE Transactions on Antennas and Propagation AP-25 104-111.

Kummerow C, Olson W and Giglio L 1996 A simplified scheme for obtaining precipitation and vertical hydrometeor profiles from passive microwave sensors; IEEE Trans. Geosci. Remote Sens. 34 1213-1232.

Liebe H J 1985 An updated model for millimeter wave propagation in moist air; Radio Science 20 1069-1089.

Marshall J and Palmer W 1948 The distribution of raindrops with size; J. Meteorol. 5 165-166.

Monahan E C and O' Muircheartaigh I G 1986 Whitecaps and the passive remote sensing of the ocean surface; Int. J. Remote Sens. 5 627-642.

Petty W G 2001 Physical and microwave radiative properties of precipitating clouds. Part I: Principal component analysis of observed multichannel microwave radiances in tropical stratiform rainfall; J. Appl. Meteorol. 40 2105-2114.

Shige S, Sasaki H, Okamoto K and Iguchi T 2006 Validation of rainfall estimates from the TRMM precipitation radar and microwave imager using a radiative transfer model: 1 . Comparison of the version- 5 and -6 products; Geophys. Res. Lett. 33 doi: 10.1029/2006GL026350.

Srinivasan J 2006 Megha Tropiques Scientific Rationale, First Workshop on Megha-Tropiques organized by Indian Space Research Organisation (ISRO), Indian Institute of Science (IISc), Bangalore.

Tao W-K and Simpson J 1993 The Goddard Cumulus Ensemble Model. Part I: Model description; Terrestrial, Atmospheric and Oceanic Sciences 4 35-72.

Tsang L, Kong J A and Shin R T 1985 Theory of Microwave Remote Sensing (New York: John Wiley \& Sons).

Ulaby F T, Moore R K and Fung A K 1981 Microwave Remote Sensing - Active and Passive (Boston: Artech House Publishers).

Varma A K, Samir Pokhrel, Gairola R M and Agarwal V K 2006 Study of geophysical parameters associated with the Orissa super cyclone using active and passive microwave remote sensing measurements; Int. J. Remote Sens. 18 3753-3765.

Viltard N, Kummerow C, Olson W S and Ye Hong 2000 Combined Use of the Radar and Radiometer of TRMM to Estimate the Influence of Drop Size Distribution on Rain Retrievals; J. Appl. Meteorol. 39 2103-2114.

Wiscombe W J 1980 Improved Mie algorithms; Applied Optics 15 1905-1909.

Wisler M M and Hollinger J P 1977 Estimation of marine environmental parameters using microwave radiometric remote sensing systems; Technical Report NRL Memo Rep. 3661, Naval Research Laboratory, Washington, DC.

Zhang G, Vivekanandan J and Politovich M K 1999 Scattering effects on microwave passive remote sensing of cloud parameters. Preprints; Eighth Conf. on Aviation, Range, and Aerospace Meteorology, Dallas, TX, American Meteorological Society, 497-501. 